\title{
Expériences sensibles de la mobilité urbaine
}

Sensory Experiences related to Urban Mobility

\section{Damien Masson}

\section{OpenEdition}

Journals

Édition électronique

URL : http://journals.openedition.org/crau/563

DOI : $10.4000 /$ crau. 563

ISSN : 2547-5746

Éditeur

Éditions du patrimoine

Édition imprimée

Date de publication : 1 novembre 2012

Pagination : 136-143

ISBN : 978-2-7577-0108-9

ISSN : 1296-4077

Référence électronique

Damien Masson, «Expériences sensibles de la mobilité urbaine », Les Cahiers de la recherche architecturale et urbaine [En ligne], 26/27 | 2012, mis en ligne le 01 novembre 2017, consulté le 02 mai 2019. URL : http://journals.openedition.org/crau/563 ; DOI : 10.4000/crau.563 
Cette recherche porte sur l'expérience de la mobilité en transports collectifs urbains et prend son origine au croisement de deux questions complémentaires. Qu'est-ce que, du point de vue de l'expérience individuelle, faire un trajet en ville? Quelles modalités de compréhension de l'espace urbain la traversée rend-elle possible? Questions qui invitent à l'ouverture de trois chantiers, méthodologique, théorique et pratique.

\section{Expériences sensibles de la mobilité urbaine}

DAMIEN MASSON

\section{Une proposition de compréhension} de l'expérience sensible des trajets et de l'urbain Dans cet article, il s'agit de restituer un travail doctoral ${ }^{1}$ sur le thème de l'expérience de la mobilité motorisée dans l'espace urbain. Cette recherche prend son origine au croisement de deux questions complémentaires : qu'est-ce que faire un trajet en ville ? Quelles modalités de compréhension de l'espace urbain la traversée rend-elle possible ? Par leur formulation, ces questions invitent à l'ouverture de trois chantiers :

- le premier, méthodologique, met en jeu la question des modes d'enquêtes et d'analyse de l'expérience individuelle et collective des trajets en transports collectifs ;

- le deuxième, théorique, vise la formulation de propositions heuristiques pour la notion d'ambiance, souvent pensée à l'échelle du corps - de l'ici et du maintenant -, plus rarement à celle de l'espace urbain (on parle volontiers d'ambiances urbaines, mais d'une ambiance urbaine... ?) ;

- le troisième, pratique, est plus liminaire et vise à rendre manifeste la portée opérationnelle (aux niveaux de la conception architecturale,

1. Damien Masson, La perception embarquée. Analyse sensible des voyages urbains, thèse de doctorat en urbanisme mention architecture sous la direction de Jean-Paul Thibaud, université Pierre Mendès-France Grenoble 2, Grenoble, CRESSON UMR 1563 «Ambiances architecturales et urbaines ", (thèse soutenue le 7 juillet 2009), 348 p. (vol. 1), et 179 p. (vol. 2).
2. Jacques Lévy, Michel Lussault (dir.), Dictionnaire de la géographie et de l'espace des sociétés, Paris, Belin, 2003, p. 989. 
urbaine et des espaces et matériels de transport) des analyses et résultats issus des deux premiers chantiers.

La restitution de ce travail prend ici la forme de courtes parties successives montrant une recherche à comprendre dans sa progression, où chaque terrain d'enquête invite à reformuler les questions d'ensemble, puis à relancer les investigations et analyses sur un mode - sensible, dans le cas présent - différent du précédent. Ainsi, du rapprochement des questions initiales, trois terrains, sur trois modalités sensorielles, et visant autant d'enjeux analytiques sont réalisés. En aucun cas, nous n'aurions su - ni tenu - à les présenter en suivant un schéma de type hypothético-déductif, qui s'il permet de donner une forme - à postériori - de stabilité à l'ensemble proposé, ne restitue pas la vivacité d'un processus de construction de résultats procédant d'une refonte successive de ses hypothèses initiales, et ne correspondrait que trop peu à ses fondements épistémologiques.

\section{La mobilité urbaine ?}

\section{Proposition d'une acception tributaire du sensible}

Les voies de définition de la ville sont légion et reflètent autant d'approches possibles sur cet " objet » ou phénomène difficile à qualifier en quelques mots. Dans une majorité de cas, des dimensions spatiales, construites et humaines restent inextricables. Par exemple, dans une option géographique, Jacques Lévy définit la ville comme « une relation spécifique entre territoires et réseaux ${ }^{2}$ ». Aussi fonctionnelle que soit cette proposition, elle sous-entend néanmoins - et de manière nécessaire - les individus qui sont, en partie, le "pourquoi » du " comment » des réseaux, supports d'allées et venues, de flux ${ }^{3}$.
L'état actuel des recherches cèderait-il de plus en plus le pas à l'« actualité » toujours grandissante des individus mobiles? En effet, le monde habité peut se comprendre à travers les notions de ville, de mobilité et de réseaux. Toutes les trois co-participent d'une nécessité - le " besoin humain de relations ${ }^{4}$ » interindividuelles -, et configurent l'expérience vécue du monde en assurant les conditions de réalisation de ce désir.

Acceptant la mobilité comme un champ recouvrant les problématiques liées à la thématique du mouvement, nous suivons la définition qu'en donne Alain Bourdin : "On appellera mobilité le fait de changer de position dans un espace réel ou virtuel, qui peut être physique, social, axiologique, culturel, affectif, cognitif ${ }^{5}$. Cette définition englobante de la mobilité rend explicite les trois modes d'appréhension du champ de la mobilité que propose Tim Cresswell ${ }^{6}$, comme : un fait brut observable empiriquement ; une représentation prenant sens à partir d'un point de vue spécifique; une pratique corporelle, une expérience. La dernière perspective proposée par Cresswell place la dimension sensible de la mobilité au premier plan. En tension entre fixité et mouvement, la mobilité procède de la gestion de la co-présence, et configure la relation personnelle au monde. En ce sens, nous considérons moins la mobilité comme un fait social explicable par des "fixités "spatiales et des motifs de tous ordres, que comme un mode possible de l'habiter, et nous suivons alors la définition qu'en donne Rachel Thomas : "De notre point de vue, la mobilité ne définit pas uniquement le processus physique par lequel I'homme se déplace et change de lieu. Elle désigne
3. Donnée quantitative, les flux « expriment des quantités et des directions, reliées à des processus de nature économique, et les trafics expriment des flux acheminés par des moyens de transport et des infrastructures organisées en réseaux ». Dans Jean-Jacques Bavoux, Francis Beaucire, Laurent Chapelon, Pierre Zembri, Géographie des transports, Paris, Armand Colin, 2005, p. 10.

4. Traduction personnelle d'une citation attribuée à Georg Simmel décrivant cette nécessité (the human will to connection) comme un point de différenciation entre humains et animaux. Dans Mimi Sheller, John Urry, "The New Mobilities Paradigm », Environment and Planning A, 2006, vol. $38, n^{\circ} 2$, p. 215. Alain Bourdin quant à lui traite de "l'irrépressible mouvement de l'individu » en analysant chez Tocqueville la nécessaire mise en relation au monde (et non à autrui comme chez Simmel) pour des fins de consommation. Dans Alain Bourdin, «Les mobilités et le programme de la sociologie ", Cahiers internationaux de sociologie, 2005, vol. 118, p. 14-15.

5. Alain Bourdin, "L'individualisme à l'heure de la mobilité généralisée ", dans Sylvain Allemand, François Ascher, Jacques Lévy (dir.), Les sens du mouvement : modernité et mobilité dans les sociétés urbaines contemporaines, (actes du colloque de Cerisy organisé par l'Institut pour la Ville en Mouvement, juin 2003), Paris, Belin, 2004, p. 92. 
plus largement une manière de mettre le passant "en prise" avec l'espace public urbain 7. " Lourde de conséquences au plan épistémique et méthodologique, cette acception de la mobilité fait de l'étude de la perception en mouvement de l'espace urbain un thème de recherche du champ de la mobilité.

\section{De la compréhension de l'expérience sensible de la mobilité urbaine à la proposition théorique de l'ambiance urbaine}

On peut faire l'hypothèse qu'il n'est pas de meilleure interface entre corps et espace urbain que le temps du mouvement. Un trajet urbain - une forme de manifestation concrète du mouvement - peut ainsi être considéré comme un entremetteur de la ville et des individus qui la traversent et est, de surcroît, une condition de possibilité de réalisation d'une expérience sensible de l'espace urbain à une grande échelle. Par là, de la même manière que Bruno Latour ${ }^{8}$ qui avance qu'il n'existe pas de distinction de nature entre le local et le global, car le global serait du local en mouvement, notre proposition ne vise pas à affirmer une différence de nature entre le sensible contextualisé et une approche sensible de l'espace urbain à grande échelle. Au contraire, il s'agit de considérer l'approche sensible à une échelle territoriale comme une réplication (prolongation, déroulement, étalement) par le mouvement du sensible localisé (contextuel, corporel, individuel).

La question, devient alors la suivante : comment élaborer un discours sensible sur la ville, qui ne se limite pas au contexte des corps, à l'espace immédiat environnant les individus, mais étant opératoire à une grande échelle ? Cette interrogation est une proposition de compréhension de l'expérience sensible qui va « au-delà de soi ", aux plans spatiaux et temporels, ayant pour objectif une tentative de "résolution " de l'oxymore qu'est une ambiance urbaine. En quoi peut-on parler de I'ambiance urbaine au singulier? Poser la question de la production d'un discours sensible sur l'espace urbain de grande échelle est une manière de tenter $d^{\prime} y$ répondre. Pour cela, la proposition méthodologique est celle de la mobilité, rendue opératoire au moyen des hypothèses suivantes:

- d'un point de vue pratique, elle permet la réalisation d'une expérience sensible de la grande échelle,

- d'un point de vue théorique, elle peut être considérée comme productrice d'une ambiance urbaine, dans la mesure où le mouvement est une condition de « mise en contact " des ambiances : situées entre l'intérieur du véhicule et l'espace traversé d'une part, et entre les différents lieux traversés d'autre part.

\section{Entre expérience sensible de la mobilité et ambiance urbaine} Le concept de "voyage urbain "

Le déploiement empirique lié à la mise en question de I'ambiance urbaine se fait ainsi dans le cadre d'un trajet en transports collectifs urbains, considéré comme un " lieu-moment ${ }^{9}$ » chargé de sens au plan de l'expérience. Afin de répondre au paradoxe des échelles, inhérent à la thématique de l'ambiance urbaine - comment comprendre les ambiances à l'échelle de la ville alors que l'ambiance environne à priori le corps ? -, nous considérons le sensible à une grande échelle comme
6. Tim Cresswell, On the Move: Mobility in the Modern Western World, Londres, Routledge, 2006, p. 3-4.

7. Rachel Thomas, sous la direction de Jean-François Augoyard, Ambiances publiques, mobilité, sociabilité : approche interdisciplinaire de l'accessibilité piétonnière des villes, thèse de doctorat en architecture/sciences humaines et sociales, université de Nantes, 2000, p. 35
8. Bruno Latour, Changer de société, refaire de la sociologie, Paris, La Découverte, 2006.
9. Complémentaire à la notion de " lieu-mouvement » proposée par Georges Amar, nous proposons celle de lieu-moment pour désigner l'espace-temps des trajets. À la différence de l'immuabilité relative des lieux-mouvements inscrits sur le sol urbain, les lieux-moments sont temporaires, il s'agit des assemblages se formant pendant le temps du mouvement concret. Ainsi, le lieu-moment n'a rien de virtuel, mais désigne l'épaisseur phénoménale et expérientielle qualifiée au cours du 
du sensible contextualisé en mouvement. D'un point de vue pratique, cela donne la construction de l'hypothèse de travail suivante, mise en jeu à tout moment de ce travail, qui consiste à considérer le trajet en ville comme un voyage urbain. Ce " concept opératoire " des ambiances ${ }^{10}$, est ainsi défini comme la caractérisation du temps du mouvement pendant lequel des ambiances de diverses natures et origines sont mises en contact pendant qu'elles sont traversées.

\section{D'un concept opératoire des ambiances aux investigations empiriques}

Ce concept permet d'orienter les investigations empiriques, en permettant de dégager une focale d'enquête spécifique relative à l'ambiance d'un trajet. Trois terrains principaux ont été menés et leur lien réside dans leur nature : il s'agit de transports collectifs ferroviaires circulant en zone urbaine dense.

- Le premier terrain répond à la question : comment qualifier l'expérience de la ville vue par la fenêtre d'un transport collectif ? Les enquêtes ont été menées à bord du train circulant entre les gares de Lyon Part-Dieu et Perrache.

- Le deuxième terrain questionne l'expérience interpersonnelle, sensible et sociale, d'un trajet à bord de la ligne 2 du métro parisien.

- Portant sur la question de la qualification sonore des traversées et en particulier la compréhension de leur rythmique, le troisième terrain, procède d'un corpus d'enregistrements sonores de traversées urbaines : dans les métros et tramways de Paris, Bonn, Varsovie, Tunis et São Paulo.

\section{Visions mobiles}

\section{Le mouvement comme révélateur des ambiances traversées}

Cette première phase de terrain qui a pour enjeu l'identification de phénomènes visuels spécifiques s'établissant entre le passager et l'environnement traversé permet la constitution d'un répertoire d'effets visuels dynamiques (flou, trilles, défilement, étagement de plans, etc. ${ }^{11}$. Ces derniers sont à la fois descriptifs et qualificatifs des modes de visualité se manifestant entre le passager et l'environnement urbain, en tenant compte de la médiation réalisée par le référentiel que constitue le moyen de transport. Le résultat majeur issu de cette typologie est que le mouvement agit comme un révélateur des ambiances situées. De fait, les effets visuels dynamiques constituent une voie d'expression de phénomènes rendus manifestes par le mouvement en qualifiant ce qui émerge d'un rapport à la ville embarqué dans un mouvement motorisé. Ainsi, ils rendent manifeste ce que l'espace des réseaux de transport contient en puissance. Nous proposons alors de considérer les formes d'appréhension de l'espace en mouvement entre : espace visible, espace visuel et espace conceptuel, ce qui permet de témoigner de la multiplicité de ses formes d'appréhension. Cette proposition l'approche pragmatiste du paysage développée par Danny Trom ${ }^{12}$, qui insiste sur le caractère à la fois partagé et individualiste du monde, en mettant l'accent sur l'existence d'un contexte physique, qui change de signification pour des personnes lui faisant face en fonction de ce qu'elles "peuvent y faire». mouvement, lequel ne pouvant ainsi plus être désigné, selon cette acception, à un "dé-placement ».

10. Nous attribuons le terme concept ou hypothèse conceptuelle au voyage urbain dans le même sens que lui donnent Deleuze et Guattari (bien qu'ils circonscrivent par ailleurs l'appartenance des concepts à la seule philosophie et non à la science) lorsqu'ils écrivent : "Tout concept renvoie à un problème, à des problèmes sans lesquels il n'aurait pas de sens, et qui ne peuvent eux-mêmes être dégagés ou compris qu'au fur et à mesure de leur solution [...] »: Gilles Deleuze, Félix Guattari, Qu'est-ce que la philosophie? $2^{\mathrm{e}}$ éd., Paris, Éditions de Minuit, 2005, p. 22.

11. Pour plus de développements à propos de ce terrain et de ce répertoire, nous renvoyons le lecteur à l'article suivant: D. Masson, «Le mouvement: un révélateur des potentialités esthétiques de l'architecture ordinaire? » dans Pascal Sanson (dir.), Les arts de la ville dans le projet urbain. Débat public et médiation, Tours, Presses universitaires François Rabelais, 2011, p. 71-86.

12. Danny Trom, «L'engagement esthétique : du trouble à l'enquête visuelle. Une pragmatique du regard sur le paysage ", dans Daniel Cefaï, Isaac Joseph (dir.), L'Héritage du pragmatisme. Conflits d'urbanité et épreuves de civisme, La Tour d'Aigues, Éditions de l'Aube, 2002. 
Certains effets visuels dynamiques montrent I'espace urbain d'une manière suffisamment spécifique pour que l'on puisse considérer le mouvement physique dans l'espace comme révélateur d'un potentiel. Par là même apparaît une propriété fondamentale du paysage : il ne peut se limiter à ses caractéristiques matérielles et, suivant Alain Roger, on peut indiquer qu'une dimension " métaphysique ${ }^{13}$ » le sous-tend. Ainsi, l'espace traversé peut être environnement, espace idéel, paysage etc. et le mouvement, orchestrant la triple relation corps, référentiel ${ }^{14}$ (ou mode de transport), espace, crée les conditions de ce changement de statut, comme en témoignent les phénomènes spécifiques se manifestant lors de la traversée.

Les effets visuels dynamiques véhiculent une possibilité de lecture renouvelée des formes urbaines, notamment à travers les anamorphoses dont ils rendent compte. Leur répertoire a pour conséquence $d$ 'asseoir une hypothèse concernant la relation entre l'environnement traversé et le passager, faisant de ce dernier une partie du paysage et non un spectateur distant. II ressort des investigations menées dans le train lyonnais la thématique du potentiel. Ce potentiel est applicable à l'espace traversé, mais également au monde phénoménal de l'expérience du mouvement. L'hypothèse du mouvement générateur de l'ambiance urbaine était probable tant que l'on accepte l'idée d'une différentiation des ambiances dans la mesure où l'ambiance urbaine procède de leur rencontre. En revanche, en indiquant la création d'un monde phénoménal spécifique basé sur un mode de relation lors de la traversée, cette hypothèse s'infléchit et le mouvement est moins compréhensible comme un générateur d'ambiance urbaine que comme un révélateur de l'ambiance urbaine contenue en puissance dans les situations locales et leurs ambiances.

\section{De la vision embarquée aux modes de relation à l'environnement}

Sur le deuxième terrain, l'enjeu visé passe de la qualification des phénomènes à ceux des modes de relation à l'environnement. En effet, l'expérience des transports collectifs est caractérisable autant au plan phénoménal qu'à celui de modalités spécifiques d'attention donnant, in fine, lieu à la production d'un répertoire de phénomènes attentionnels. Cette entreprise tâche de décrire et de comprendre, par le truchement des effets attentionnels, les rapports qu'entretiennent les passagers avec l'environnement spatial, temporel, sensible et social de l'espace technique et urbain du réseau métropolitain parisien. Le point de départ individualiste configure fortement ces effets sensibles qui concernent prioritairement des modalités d'inscription corporelle et psychologique au sein du déroulement du trajet. Sont ainsi repérés et décrits, des régimes d'attention, des tonalités affectives et des conduites.

La majorité des effets attentionnels caractérisent un rapport sujet-contexte de type médial, c'est-àdire : là où se jouent les interactions et échanges, que ce soit avec les autres ou le monde ${ }^{15}$. Les effets visuels dynamiques ne caractérisent jamais ce type de relation, en raison de l'impossibilité d'action vis-àvis du territoire traversé, lequel est selon le vocable

13. Alain Roger, Court traité du paysage,

Paris, Gallimard, 1997.

14. Ferdinand Gonseth, Le référentiel, univers obligé de la médiatisation, Lausanne, L'Âge d'homme, 1980. 
d'Amphoux soit environnement, soit paysage. Ainsi, se spécifie une différence majeure entre les deux répertoires. Surtout, elle souligne une dimension fondamentale du voyage urbain : en mouvement, le rapport visuel au monde traversé est de type environnemental ou paysager tandis que le rapport au référentiel (contexte social et matériel immédiat) est de type médial. Alors, les éléments de définition du concept de voyage urbain avancent dans la mesure où la mise en relation d'ambiances différenciées dont il procède est remarquable en termes de rapport au monde. Un voyage urbain se caractérise ainsi par le moment où le passager entretient plusieurs modes de rapport au monde simultanément. Par exemple, en voyage urbain le passager peut être : embarqué dans un milieu et mouvant dans l'environnement ou le paysage.

Par ailleurs, à l'interface des deux répertoires apparaissent des formes de temporalisation spécifiques. Tous les effets en question mettent en œuvre des temporalités différenciées et le voyage urbain se qualifie alors au plan temporel à la rencontre de deux temporalités : phénoménale et attentionnelle. Le troisième terrain d'enquête vise alors l'investigation de cette thématique.

\section{Écoutes voyageuses}

\section{Vers une rythmique du voyage urbain}

Le terrain sonore met l'accent sur la thématique temporelle des voyages urbains, abordés à travers la notion de rythme. La dynamique temporelle du voyage urbain est ainsi considérée comme une tension entre un niveau de fond et un niveau d'émergence, située au croisement des temporalités d'attention et des temporalités propres aux phénomènes. Les résultats issus de cette partie concernent autant le matériau sonore même que la compréhension du trajet puis celle des ambiances. Quatre "référents sonores » types - constructions caractéristiques du monde audible des trajets, qualifiant à la fois des signaux sonores et leurs modes d'ajustement temporels - des transports collectifs apparaissent, en étant descriptifs :

- du dispositif constitué par le système de transport : élargissement de l'enveloppe acoustique (à l'arrêt), vague phonique (en mouvement), portes et sonneries (entre l'arrêt et le mouvement ) ;

- des dispositions prises par les usagers dans ce contexte spécifique : situation de composition ${ }^{16}$ comme pratique usagère nécessaire.

Ces quatre référents sont constitutifs de la dynamique des ambiances dans la mesure où leur construction participe de l'agencement des plans de fond et d'émergence se co-définissant. À partir de la caractérisation de ces référents descriptifs des traversées urbaines sonores, une entreprise de description sonore de six trajets urbains spécifiques est construite. La typologie qui en ressort permet de dégager quatre modes spécifiques d'articulation du fond et des émergences comme dimensions constitutives de l'ambiance urbaine : émergences dominantes, fond dominant, instabilité métabolique, co-constitution. Enfin, de ces modes d'articulation du fond et des émergences deviennent manifeste deux formes spécifique d'organisation de la temporalité inhérente à l'ambiance de trajets :
15. Pascal Amphoux, «Environnement, milieu et paysage sonores ", dans Michel Bassand, Jean-Philippe Leresche (dir.), Les faces cachées de l'urbain, Berne, Peter Lang, 1994, p. 159-176.
16. L'article suivant développe particulièrement la question des ajustements sensibles et sociaux, caractéristiques de la « pragmatique usagère » en situation de transports : D. Masson, « Musique à bord! Transports collectifs et expression musicale », Les Cahiers du Gerhico n 13, "Accords et à cris. Études pluridisciplinaires sur la sonorité », Poitiers, MSHS, 2009, p. 75-86. 
- la trajectoire inexorable : qualifiant un mode de rapport au temps de type linéaire,

- le cycle : caractérisant une forme perçue liée à la répétition.

Ces deux modes d'appréhension de la temporalité des trajets permettent d'avancer sur la thématique de la rythmique spécifique des voyages urbains, constituée a minima par la répétition d'une structure phénoménale spécifique : la séquence entre deux stations, laquelle procède à la fois de la trajectoire (un trajet constitue un mouvement continu d'un endroit à un autre) et du cycle (un trajet active un ensemble de phénomènes répétitifs : accélération, roulement, décélération, arrêt, ouverture des portes, sonnerie, fermeture des portes etc.)

Manifeste au plan sonore, cette structure caractérise la forme du mouvement en transports collectifs et joue sur un ensemble de modalités sensibles : kinesthésique en premier lieu, mais aussi visuelle, olfactive, thermique etc. Cette séquence est de nature temporelle : elle est répétitive. Ainsi, indiquer ce qui pourrait sembler ressortir de l'évidence (un trajet est une succession de phases d'arrêt et de mouvement) engendre un ensemble de conséquences pour la compréhension de l'expérience du trajet en ville : faire l'expérience d'un trajet n'est pas seulement relier deux places distinctes dans l'espace ; faire l'expérience du trajet n'est pas uniquement rejoindre le système technique, effectuer une traversée, quitter le système technique. La réalisation d'un trajet en transports collectifs commence par l'expérience d'une durée qualifiée par la répétition de la séquence sonore (et sensible, par extension) entre deux stations. Le premier degré de qualification du voyage urbain est d'ordre rythmique : un temps qualifié par la répétition d'une séquence de phénomènes. Condition de possibilité de cette répétition, l'espace qui cadre le mouvement et concoure à donner le tempo de ce rythme cyclique. Ainsi, en observant le rythme instauré en voyage urbain, c'est l'espace du trajet où se fait l'ambiance qui peut être observé et compris.

\section{Une conclusion en forme de programme}

À l'issue de ces investigations, l'interprétation croisée de l'ensemble des résultats empiriques nous conduit à affirmer que le voyage urbain est constitutif d'une ambiance urbaine à la fois continue et plastique, caractéristique d'une construction rythmique propre. Expérience sensible de la grande échelle, le voyage urbain donne accès à l'ambiance urbaine en tant que configuration rythmique des ambiances. Par exemple, en trajet, la séquence " entre deux stations" se répète cycliquement et engendre un ensemble de phénomènes, remarquables aux plans sociaux, sensibles et construits. La progression du trajet dans le temps et dans l'espace urbain multiplie les occurrences phénoménales qui ajoutent à leur tour d'autres modes temporels. Ainsi, en mouvement motorisé, une dynamique d'unification se met à l'œuvre pour composer et affecter temporairement une ambiance de trajet se composant phénoménalement de l'évolution d'un rapport de fond à émergences, organisé rythmiquement. Nous formulons alors la proposition suivante : l'ambiance urbaine correspond au réagencement des ambiances situées sur un mode spécifique qui est celui du temps polyrythmique. La polyrythmie est la manière 
par laquelle les phénomènes et, plus généralement, les " composantes " du voyage urbain sont mises en relations. Ces dernières correspondent aux registres analytiques mis en place tout au long de ce travail. Autrement dit : un voyage urbain correspond à la mise en relation des individus, du référentiel et de l'espace urbain et cette interrelation spécifique configure un ensemble de rapports convoquant des dimensions sociales, paysagères, imaginaires, construites et temporelles, organisées en de multiples temporalités, ou bien : un temps polyrythmique. L'ambiance urbaine serait ainsi le produit de cette organisation : elle est une configuration rythmique des ambiances.

S'ouvre par là même tout un programme de travail, sur l'échelle des ambiances et les temporalités des espaces urbains, lesquelles, dans une perspective rythmanalytique, ne seraient plus seulement résultantes de phénomènes localisés, mais bien constitutives de "l'idée de ville». 